\title{
MiR-429 Determines Poor Outcome and Inhibits Pancreatic Ductal Adenocarcinoma Growth by Targeting TBK1
}

\author{
Bin Song Kailian Zheng Hongyun Ma Anan Liu Wei Jing Chenghao Shao \\ Gang Li Gang Jin
}

Department of Pancreatic Surgery, Changhai Hospital, Second Military Medical University, Shanghai, China

\section{Key Words}

MiR-429 • PDAC • MyD88 • Patients survival • Cancer growth

\begin{abstract}
Background: Pancreatic ductal adenocarcinoma (PDAC) ranks fourth on the list of cancerrelated causes of death and its prognosis has not improved significantly over the past decades. Deregulation or dysfunction of miRNAs contribute to cancer development. Previous data indicates that miR-429 is involved in the pathogenesis of PDAC. However, the role of miR-429 in PDAC remained unknown. Methods: MiR-429 levels in sample tissues of 78 patients and in PANC1 and SW1990 cell lines were quantified by real-time PCR. MiR-429 expression was modulated using specific pre- and anti-miRNAs and cell growth was assayed by MTT analysis. Bioinformatics prediction of the miR-429 putative target genes was performed and luciferase assays confirmed TBK1 as a direct target gene. TBK1 levels in PDAC tissues were analyzed by immunohistochemistry. Results: MiR-429 was remarkably decreased in PDAC tissues and cell lines. Lower miR-429 expression in PDAC tissues significantly correlated with shorter survival of PDAC patients. Overexpression of miR-429 inhibited PDAC cell lines growth in vitro and vice versa. TBK1 was found to be the direct target gene of miR-429. Higher TBK1 protein level in PDAC tissues correlated with shorter survival of PDAC patients. Overexpression of TBK1 partly restored cell proliferation. Conclusions: Low level of miR-429 and high level of TBK1 in PDAC promoted PDAC cells growth which might be related to the low survival rate of PDAC patients. MiR-429 play its role in PDAC by targeting TBK1.
\end{abstract}

B. Song, K. Zheng and H. Ma contributed equally to this work.

Gang Li

and Gang Jin

KARGER 125
Department of Pancreatic Surgery, Changhai Hospital, Second Military Medical University, NO.168 Changhai Road, Shanghai, 200082 (China)

Fax+86-15-2914-1717, E-Mail li_gang169@163.co, E-Mail jingangjinggang@126.com 


\section{Introduction}

Pancreatic ductal adenocarcinoma (PDAC) ranked 13th on the list of most commonly diagnosed cancers and was the fifth most common cause of cancer death in China in 2006 [1].The prognosis of PDAC has not improved significantly over the past decades. Surgical resection, for which only a minority $(<20 \%)$ of patients qualify due to advanced stage of disease at the time of diagnosis, is currently the only chance for cure. Surgical resection was found to improve five-year survival rates from $<4 \%$ if left untreated to $25-30 \%$ after resection [2,3]. Due to the fact that cancer is a complex disease involving numerous genetic and epigenetic changes, a better understanding of the molecular mechanism of PDAC is urgently needed to develop an effective diagnosis and treatment.

MiRNAs are a class of small RNAs approximately 18-22 nucleotides in length. MiRNAs suppress protein expression by inhibiting translation or inducing mRNA degradation by binding to the 3'-untranslational region (3'UTR) of target mRNAs [4]. Deregulation or dysfunction of miRNAs contribute to cancer development [4-9]. The miRNAs expression profiles in pancreatic tumor tissues were different from those identified in a normal pancreas or in chronic pancreatitis [10]. MiRNAs were also involved in the pathogenesis of PDAC. For example, the miR-200 family appeared to have important roles in PDAC and have been shown to regulate epithelial to mesenchymal transition by targeting ZEB1 and SIP1 [11, 12].

Mees et al. have classified 16 human PDAC cell lines into three hierarchical groups according to their metastatic potential, and profiled their mRNA and miRNAs expression. The highly metastatic PDAC cell lines, when compared to the non-metastatic cell lines, have shown decreased mRNA and protein expression of EP300, which is related to significant up-regulation of EP300-targeting mRNAs (miR-194, miR200b, miR-200c and miR-429) [13].

In the study, we focused on the role of miR-429 in PDAC. We found lower miR-429 level in 78 PDAC tissues correlated with low patients survival rate. Next, we investigated the role of miR-429 by up or down regulation of miR-429 levels in PDAC cell lines. At this point, the target genes of miR-429 were predicted. TANK binding kinase 1 (TBK1) was selected for further study because it has been shown to play an important role in angiogenesis, transformation, and cell survival $[14,15]$. However, the biological function of TBK1 in PDAC remains unknown. TBK1.

In all, our data showed that low level of miR-429 in PDAC promoted PDAC growth via

\section{Materials and Methods}

\section{Patients and sample}

Surgical specimens from 78 PDAC patients and matched tumor-adjacent normal tissues were obtained postoperatively in 2009 from the Department of General Surgery, Changhai Hospital, Second Military Medical University (Shanghai, China). All patients gave signed, informed consent for their tissues to be used for scientific research. Ethical approval for the study was obtained from Changhai Hospital, Second Military Medical University (Shanghai, China). All diagnoses were based on pathological and/or cytological evidence. The histological features of the specimens were evaluated by senior pathologists according to the World Health Organization classification criteria. Tissues were obtained prior to chemotherapy and radiotherapy and were immediately frozen and stored at $-80^{\circ} \mathrm{C}$ prior to qRT-PCR assay. Matched tumor-adjacent normal tissues were used as control. 78 patients had been followed-up for 50 months and complete clinical data was electronically recorded. Readers can acquire the clinical information via E-mail jingangjinggang@126. com.

Cell culture and reagent

HEK293, PANC1 and SW1990 were obtained from the Cell Bank of Chinese Academy of Science (Shanghai, China) and cultured in DMEM medium (Hyclone, South Logan, UT, USA) supplemented with 10\% fetal bovine serum (Hyclone), $2 \mathrm{mM}$ L-glutamine and $100 \mu \mathrm{g} / \mathrm{mL}$ penicillin/streptomycin (Bio Light, 
Shanghai, China) as described in previous studies $[16,17]$. Amlexanox were obtained from Shengong campany (Shanghai, China).

Quantitative real-time PCR ( $q R T$-PCR)

The qRT-PCR analysis for miR-429 was performed by Shengong Company (Shanghai) using standard protocols on an Applied Biosystem's 7500 HT sequence Detection System. This assay includes a reversetranscription step performed using a High-Capacity complementary DNA Archive Kit (Applied Biosystems), in which a stem-loop reverse-transcription primer specifically hybridizes with an miRNA and then is reversetranscribed with MultiScribe reverse transcriptase.miR-429 expression was assessed using a mirVana ${ }^{\mathrm{TM}}$ qRT-PCR miRNA Detection Kit (Ambion, USA). The primers were designed and synthesized by Shengong Company (Shanghai, China). miR-429 sense strand UAAUACUGUCUGGUAAAACCGU; miR-429 antisense strand CAAGAUCGGAUCUACGGGUUUU; NC sense strand UUCUCCGAACGUGUCACGUTT; NC antisense strand ACGUGACACGUUCGGAGAATT. MiRNA-U6 was used as an internal control.

MTT assay

For MTT assay, $5 \times 10^{3}$ cells per well were seeded in triplicate in a 96-well plate with complete growth medium. The viability of the cells was measured over 5 days using the MTT assay (Promega, Fitchburg, WI, USA) as described previously [7, 18, 19]. The data was read by Microtiter plate reader 570-nm filters (Promega, Fitchburg, WI, USA).

MiRNAs antisense, miRNAs mimics, oligonucleotides, siRNA and pcDNA3.1-TBK1

MiRNAs mimics (miR-429 mimics) and miRNAs antisense oligonucleotides (miR-429-ASO) were obtained from GenePharma (GenePharma, China). MiRNAs ASO, miRNAs mimics, and negative control (NC) were transfected into cells at a concentration of $50 \mathrm{nM}$ using Lipofectamine 2000 (Invitrogen, Canada) transfection reagent according to the manufacturer's instructions. $48 \mathrm{~h}$ later, the cells were collected for further experiments [20]. siRNA and pcDNA3.1-TBK1 were designed by standard methods by Shenggong company (Shanghai, China) according to previous studies [21, 22].

Bioinformatic prediction of putative targets for miR-429

TargetScanHuman (http://www.targetscan.org/vert_61/) [23-26] is applied to identify the potential target of miR-429.

TBK1 3'UTR reporter analysis

The TBK1 3'UTR reporter plasmids (pRL-TBK1) were constructed by Shengong Company (Shanghai, China). Mutation in the miR-429 seed regions of the TBK1 3'UTR were generated using QuikChang Multi site -directed mutagenesis kit (Promega, Fitchburg, WI, USA). RL reporter plasmids (3.6fmol) and pGL3control (500ng for normalization; Promega, Fitchburg, WI, USA) were transfected with Lipofectamine 2000 (Invitrogen, Canada) into HEK293 $\left(6 \times 10^{4}\right.$ cells per well). Cells were collected after $48 \mathrm{~h}$ for assay using the Dual Luciferase reporter assay system (Promega, Fitchburg, WI, USA) [27].

\section{Western blot and antibodies}

Tumor tissues were collected, lysed, and blotted as described previously [6]. Membranes were blocked with blocking solution (5\% skim milk in TBST) and incubated with primary antibody, followed by the incubation with appropriate HRP-conjugated secondary antibody. The TBK1 antibody (anti-TBK1) was purchased from Santa Cruz Biotechnology, Inc [28]. The densitometry of Western blot results was measured using ImageJ software.

\section{Immunohistochemistry}

Briefly, 4- $\mu \mathrm{m}$ thick sections were cut and anti-TBK1 antibody [28] (Santa Cruz, CA, USA) was applied. Subsequent counterstaining was performed with hematoxylin. Immunostaining results for TBK1 were evaluated using a semi-quantitative scoring system, which was previously described [29], which calculated the staining intensity and the percentage of positive cells. IHC staining was scored according to the following criteria:,$- 0-10 \%$ of the nucleated cells stained,,$+ 10-40 \%$ stained, $++; 40-70 \%$ stained and,$+++ 70-100 \%$ stained. TBK1 expression was considered to be observed when score $\geq+$. Alternatively, IHC score of TBK1 expression was $(-\sim+)$ and $(++\sim+++)$, which represented low and high expression, respectively. 
Fig. 1. Low miR-429 level in PDAC tissues was correlated with low survival rate. The miR-429 expression in 78 pairs PDAC and in matched tumor-adjacent normal tissues were assayed by qRT-PCR. The difference of miR-429 expression between PDAC and in matched tumor-adjacent normal tissues were compared (A). Box-plot of miR-429 expression in 78 pairs PDAC and matched tumor-adjacent normal tissues, the mean expression of miR-429 in 78 pairs PDAC and matched tumor-adjacent normal tissues

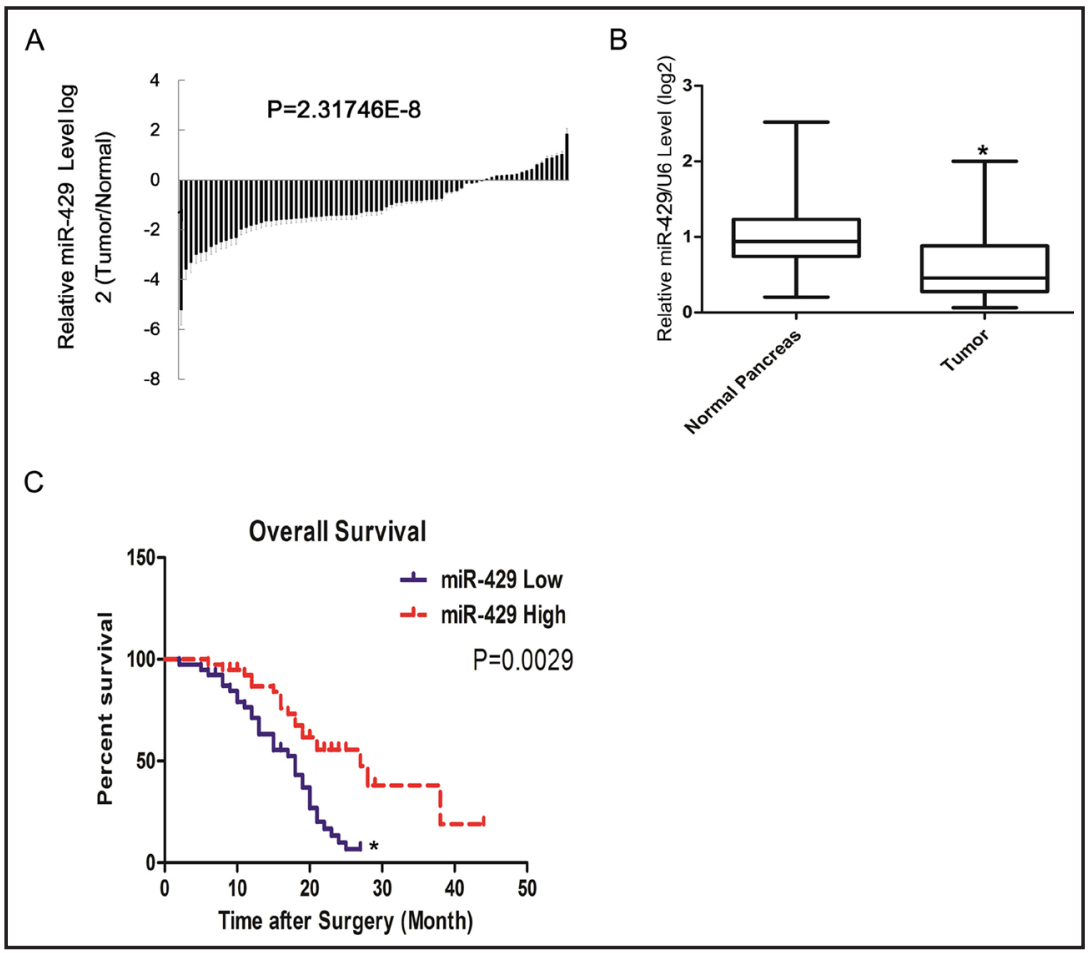
were compared (B). The Kaplan-Meier plot of overall survival in PDAC patients post-operation according to the expression of miR-429 (C). All qRT-PCR experiments were performed for three times. Data are mean \pm s.d. of three separate experiments. $* \mathrm{P}<0.05$.

TBK1 mRNA expression in PDAC analysis

The data of TBK1 mRNA in PDAC was queried from Gene Expression Omnibus (GSE15471).

\section{Statistical Analysis}

Data was presented as the mean \pm s.d from at least three independent experiments. The difference between the groups was analyzed using two-tailed Student's $t$ test when only two groups were compared. The difference between the groups was analyzed using ANOVA when three or more groups were compared. The Wilcoxon matched-pairs signed rank test was used to determine if there was a statistically significant difference in the expression of miR-429 between matched pairs. A two-tailed Mann-Whitney U test was used to determine if there was a statistically significant difference in the expression of miR-429 between any two groups. Correlation analysis was performed by two-tailed Pearson's correlation coefficient analysis. Patients survival was determined by The Kaplan-Meier analysis. Statistical analyses were performed using SPSS software (version 17.0). $\mathrm{P}<0.05$ was considered significantly different.

\section{Results}

Expression of miR-429 in PDAC

Initially, the miR-429 level in PDAC tissues and matched tumor-adjacent normal tissues were assayed by qRT-PCR. We found in the 61 of 78 pairs, miR-429 level in PDAC tissues were lower than tumor-adjacent normal tissues (Fig. 1A). The mean level of miR-429 was lower in PDAC tissues than in matched tumor-adjacent normal tissues (Fig.1B). To evaluate the clinical significance of miR-429 in PDAC, we investigated whether the level of miR-429 was associated with overall survival in PDAC patients. 78 PDAC patients have been followedup for 50 months. The median value miR-429 in all 78 case was chosen for the cutoff point for separating miR-429 high expression tumors $(\mathrm{n}=39)$ from miR-429 low expression case $(\mathrm{n}=39)$. Survival curve showed that patients with high miR-429 levels (39 cases) had a 


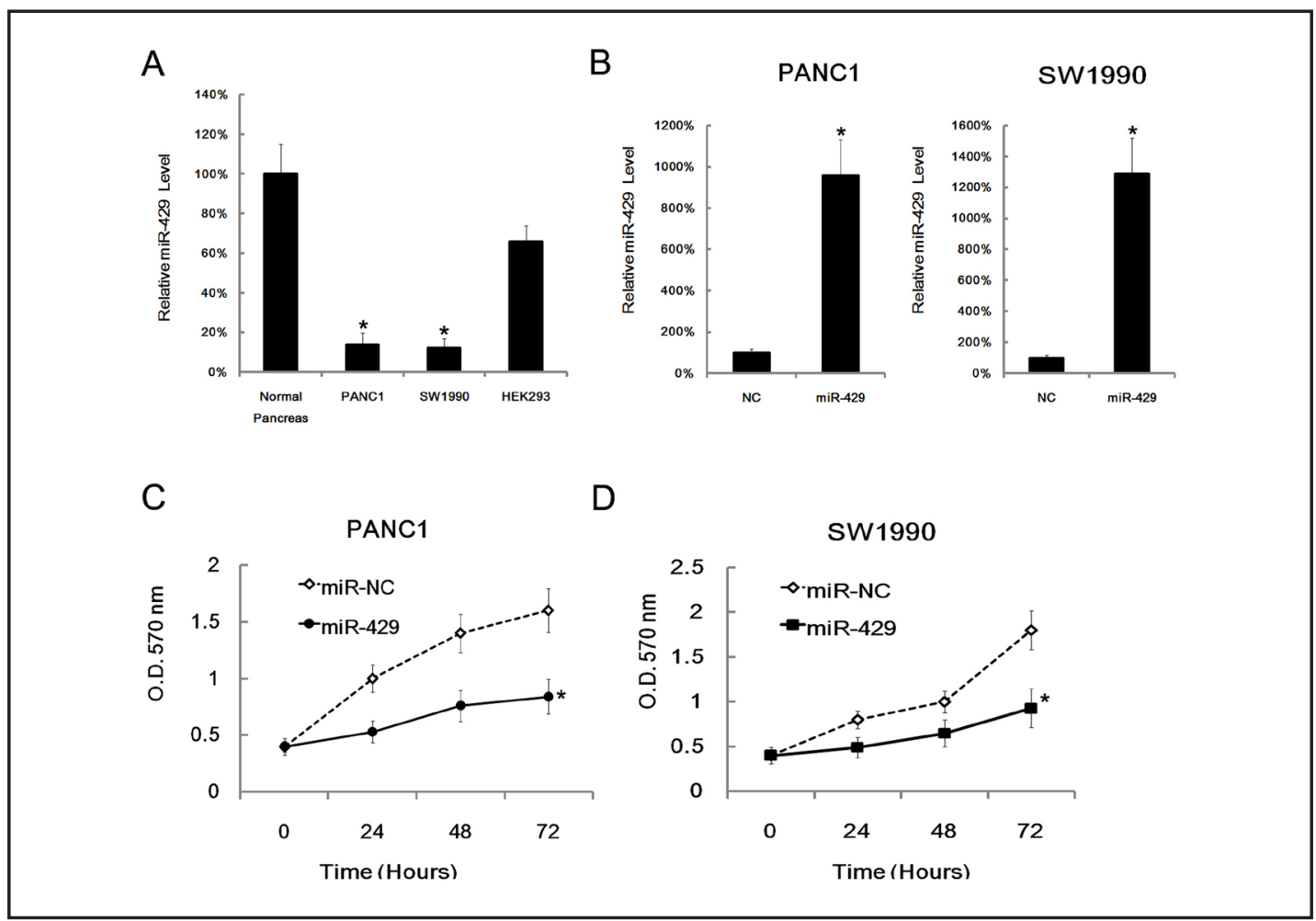

Fig. 2. MiR-429 mimics transfection inhibited PDAC cell lines growth. The miR-429 levels in normal pancreas tissues, PANC1, SW1990 and HEK293 cell lines were assayed by qRT-PCR. The miR-429 expression in normal pancreas tissue was arbitrarily defined as 100\% (A). $48 \mathrm{~h}$ after miR-429 mimics transfection, the level of miR-429 in PANC1, SW1990 cell lines were assayed by qRT-PCR. The miR-429 expression in normal pancreas tissue was arbitrarily defined as $100 \%$ (B). $48 \mathrm{~h}$ after miR-429 mimics transfection, PANC1 proliferation was assayed by MTT analysis at the indicted time points (C). Similarly, SW1990 proliferation was assayed by MTT (D). All data are mean \pm s.d. of three separate experiments. ${ }^{*} \mathrm{P}<0.05$.

significantly longer overall survival than patients with low miR-429 levels (39 cases) (Fig. 1C).

Up-regulation of miR-429 inhibited PDAC cell lines growth

We then investigated the role of miR-429 in PDAC cell lines. We initially measured the miR-429 level in PANC1 and SW1990 cell lines. We found the level of miR-429 in PDAC cell lines was lower than in normal pancreas and HEK293 cell lines (Fig. 2A). Next we upregulated the level of miR-429 by miR-429 mimics transfection in PANC1 and SW1990 cell lines. The effectiveness of transfection was verified by qPCR (Fig. 2B). $48 \mathrm{~h}$ after mimics transfection, cellular proliferation was assayed by MTT analysis. We found that up-regulation of miR-429 inhibited PANC1 and SW1990 cells proliferation.

Down-regulation of miR-429 promoted PDAC cell lines growth

We then down-regulated the level of miR-429 by miR-429 ASO (anti-miR-429) transfection in PANC1, SW1990 and HEK293 cell lines. We found miR-429 ASO transfection down-regulated the miR-429 level in the three cell lines. As PANC1, SW1990 cell lines showed a low level of miR-429, miR-429 ASO transfection did not show dramatic effects (Fig. 3A). Then cellular proliferation was assayed by MTT analysis. We found miR-429 ASO transfection mildly promoted cells growth in PANC1, SW1990 cells (Fig. 3B, C), and greatly promoted cells growth in HEK293 cells (Fig. 3D). 




Fig. 3. MiR-429 ASO transfection promoted PDAC cell lines growth. PANC1, SW1990 and HEK293 cell lines were transfected with miR-429 ASO. 48 h later miR-429 levels in normal pancreas, PANC1, SW1990 and HEK293 cell lines were assayed by qRT-PCR. The miR-429 levels in normal pancreas cells were arbitrarily defined as 100\%. The relative expression of miR-429 in PANC1, SW1990 and HEK293 after miR-429 ASO transfected were shown (A) . 48 h after miR-429 mimics transfection, PANC1 (B), SW1990 (C) and HEK293 (D) cell line proliferation was examined by MTT assay at the indicted time points. All data are mean \pm s.d. of three separate experiments. ${ }^{*} \mathrm{P}<0.05$.

TBK1 was targeted by miR-429

To explore the potential target genes of miR-429, we applied bioinformatics algorithms to predict. Many genes were predicted including TANK binding kinase 1 (TBK1) (Fig. 4A). TBK1 is a noncanonical IкB kinase (IKK) family member that mediates the innate immune response [30]. For cancer, emerging evidence demonstrates that TBK1 plays an important role in angiogenesis, transformation, and cell survival $[14,15]$. Therefore, TBK1 was chosen for further investigation. The binding sites and mutated sites in TBK1 are shown in Fig. 4A. 3 'UTR and its mutated version of TBK1 were cloned into luciferase reporter plasmids. MiR429 (or anti-miR-429) and the reporter plasmids were co-transfected into HEK293 cells. We found that in wild type 3' UTR, miR-429 reduced the luciferase activity; anti-miR-429 increased the luciferase activity, while in mutated version, the difference between experiment group and control was not significant (Fig. 4B). Consequently, we tried to confirm the relation between miR-429 and TBK1 in protein level. Data from Western blot showed that $48 \mathrm{~h}$ after miR-429 mimics transfection, TBK1 protein level was inhibited. Therefore, this data indicates that TBK1 was targeted by miR-429 (Fig. 4C).

High TBK1 protein level in PDAC tissues was correlated with low survival rate

To investigate the role of TBK1 in PDAC, the TBK1 level in 78 PDAC tissues and matched tumor-adjacent normal tissues were examined by IHC. Six representative cases were shown. The first three cases showed significantly higher TBK1 expression (Fig. 5A). In 78 pairs of PDAC tissues and matched tumor-adjacent normal tissues, there were 66 of 78 pairs with 
A
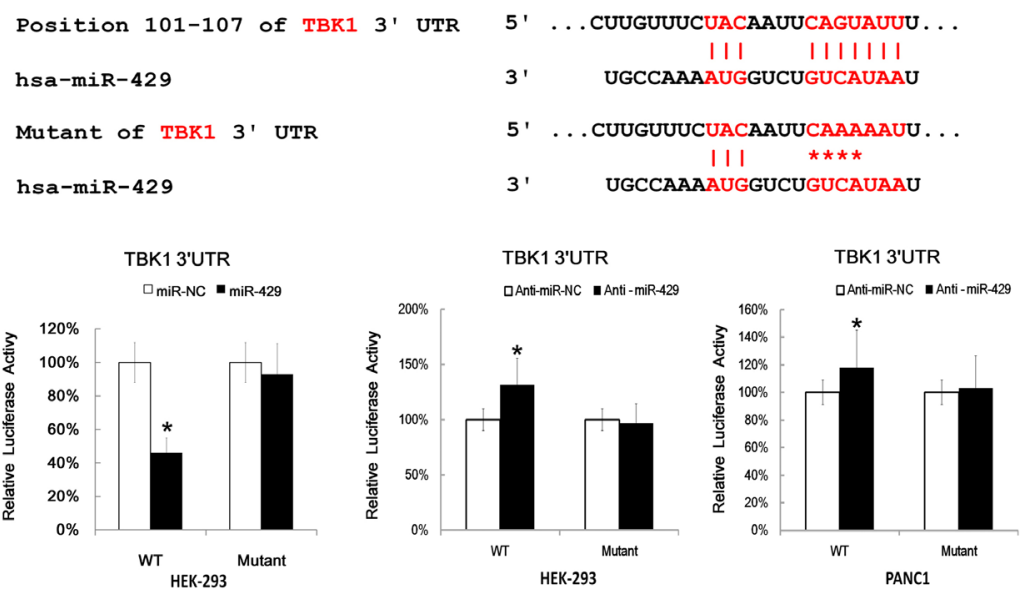

C

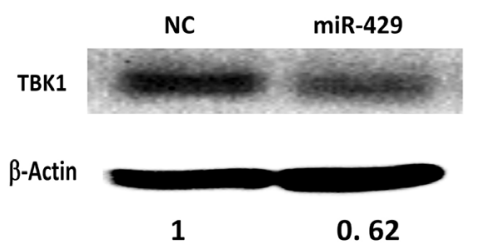

Fig. 4. Target genes of miR-429 were predicted and confirmed. Putative targeted genes were predicted by TargetScanHuman, the binding sites of putative targeted gene, and mutated sites of miR-429 were shown (A). The RL reporter plasmids (RL-control, RL- TBK1, RL-mutated TBK1) and miR-429, anti-miR-429 or control were co-transfected into HEK293 cells, along with a firefly luciferase reporter (pGL control) for normalization. Luciferase activities were measured after $48 \mathrm{~h}$. Then the ratio of RL activity of firefly luciferase activity in miR-429 treated group were calculated and compared with the ratio in miR-NC group (which was arbitrarily defined as 100\% ) (B). $48 \mathrm{~h}$ after miR-429 mimics transfection, TBK1 levels were assayed by Western blotting (C). Data are mean \pm s.d. of three separate experiments or representative image of at least three separate experiments. ${ }^{*} \mathrm{P}<0.05$.

higher TBK1 level in PDAC tissues than in normal control (Fig. 5B). We calculated the mean expression of the 78 PDAC tissues, and found the mean expression of TBK1 in PDAC tissues was higher than the matched tumor-adjacent normal tissues (Fig. 5C). To evaluate the clinical significance of TBK1 over-expression in PDAC, we investigated whether the levels of TBK1 were associated with overall survival in PDAC. 78 PDAC patients were followed up for 50 months. The Kaplan-Meier curves indicated that patients with high TBK1 expression had a significantly shorter overall survival $(p=0.0015)$ than those with low TBK1 expression. The median value of all 78 case was chosen as the cutoff point for separating TBK1 high expression cases from TBK1 low expression cases (Fig. 5D). Next we explored Gene Expression Omnibus (GEO) for analysis of TBK1 mRNA expression in PDAC. We found TBK1 expression in tumor tissues were higher than in adjacent normal tissue (GSE15471) $\left(p=5.7 \times 10^{-8}\right)$ (Fig. 5E). Next we investigated the correlation between miR-429 and TBK1, we found there were only 18 of 78 patient with high miR-429 level, and about $70 \%$ of the sample with high TBK1 level showed low miR-429 levels. And as expected, the TBK1 levels and miR-429 levels in the 78 samples was negative correlated (Fig. 5F). 
Fig. 5. High TBK1 protein level in PDAC tissues was correlated with low survival rate. TBK1 protein levels in 78 pairs of PDAC tissues and matched tumor-adjacent normal tissues were assayed by IHC. Six representative cases were shown (A). TBK1 protein levels in 78 pairs of PDAC tissues and matched tumor-adjacent normal tissues were compared (B). The mean level of TBK1 in the 78 pairs (C). The Kaplan-Meier plot of overall survival in PDAC patients post-operation according to the expression of TBK1 (D). TBK1 expression in PDAC tissues were higher than in adjacent normal tissue (GSE15471) (E). The 78 samples were divided into two groups (miR-429 high versus miR-429 low) by the median value, the correlation between miR-429 and TBK1 was calculated (F). Data are mean \pm s.d. of three separate experiments. ${ }^{*} \mathrm{P}<0.05$.

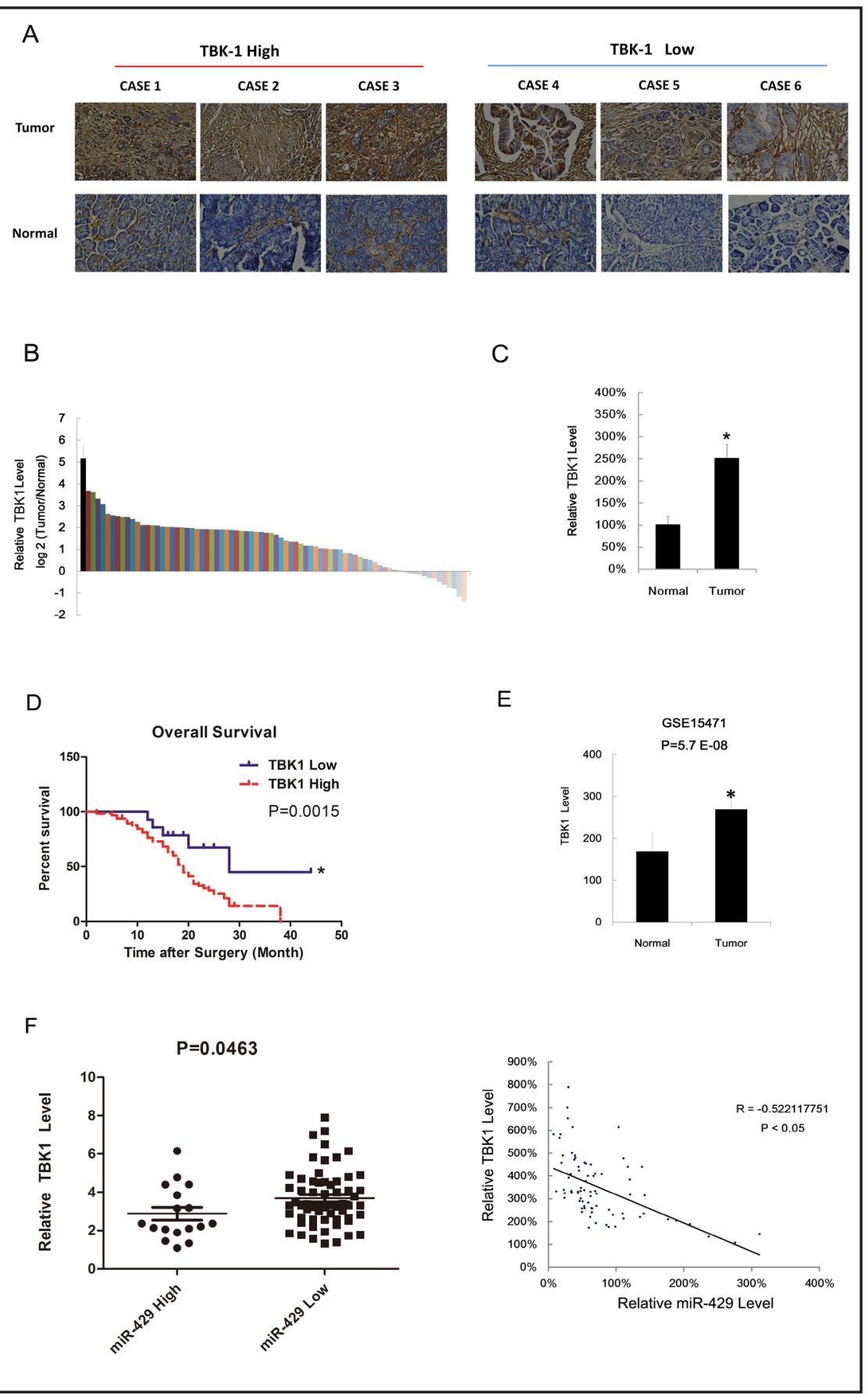

Down-regulation of TBK1 reduced cell proliferation ability and overexpression of TBK1 restore the cell proliferation ability

To further confirm the role of TBK1 in PDAC, we down-regulated the TBK1 expression in PANC1 and SW1990 by siRNA targeting TBK1 (si-TBK1) and amlexanox. Amlexanox is a specific inhibitor of TBK1 [28]. $48 \mathrm{~h}$ after siRNA transfection and $24 \mathrm{~h}$ after amlexanox treatment, we found that si-TBK1 and amlexanox both reduced the PANC1 and SW1990 cell viability (Fig.6A). Cell proliferation revealed by MTT analysis also confirmed the inhibitory effect of si-TBK1 (Fig. 6B). Next we performed the rescue experiment by transfecting TBK1 overexpression plasmid (pcDNA.3-TBK1, abbr. p-TBK1). We found that p-TBK1 transfection 




Fig. 6. Down-regulation of TBK1 reduced cell proliferation ability and overexpression of TBK1 restore the cell proliferation ability. PANC1 and SW1990 cells were treated with si-TBK1 transfection and amlexanox $(1 \mu \mathrm{M}), 48 \mathrm{~h}$ after siRNA transfection and $24 \mathrm{~h}$ after amlexanox treatment, cell viability was assayed by MTT (A). After si-TBK1transfection, PANC1 and SW1990 cells proliferation was assayed by MTT at the indicted time points (B). PANC1 and SW1990 cells were treated miR-429 mimics transfection and p-TBK1 transfection separately or co-transfection of miR-429 and p-TBK1, then the cells proliferation was assayed by MTT at the indicted time points $(\mathrm{C})$. Data are mean \pm s.d. of three separate experiments. ${ }^{*} \mathrm{P}<0.05$.

can partly restore the cell proliferation ability as comparing with only miR-429 treated group (Fig. 6C).

\section{Discussion}

In this study, we found that low miR-429 level and high TBK1 protein in PDAC tissues both correlated with low survival rate. We found that up-regulation of miR-429 inhibited PDAC cell lines growth and vice versa. 3'UTR of TBK1 was targeted by miR-429. Therefore, we concluded that low miR-429 level in PDAC promoted tumor cells growth which contributed to the low PDAC patients survival rate. It was also revealed that miR-429 exerted its role by targeting TBK1.

Our data highlights the role of miR-429 in the growth of PDAC cells. Our finding may be the first time to reveal the role of miR-429 in PDAC. It was apparent that low miR-429 promoted PDAC growth which contributed low survival rate of PDAC patients. To confirm this causal chain, further in vivo experiments will be required. MiR-429 was prove to be down-regulated in many cancers $[31,32]$. These findings coincide favorably with our data. As an interesting incidental finding, the miR-429 expression in endometrial carcinoma and bladder cancer were also up-regulated [33, 34]. In addition, higher expression levels of miR429 was correlated with a poor prognosis in patients with serious ovarian carcinoma [35]. The different role of miR-429 was possibly due to the fact that miRNAs could down-regulate numerous targets, including oncogenes and tumor suppressor genes.

Here we focused on the effect of miR-429 on growth, however, miR-429 showed multiple functions in other studies. Previous studies have shown that ectopic overexpression of miR429 in mesenchymal-like ovarian cancer resulted in reversal of the mesenchymal phenotype (mesenchymal-epithelial transition, MET) [36]. Therefore, other potential roles of miR-429 in PDAC needed further investigation. 
Our data showed that high TBK1 protein in PDAC tissues was correlated with low survival rate. The reasons behind this correlation will require further investigation. TBK1 plays a central role in innate immunity: TBK1 acts as an integrator of multiple signals induced by receptor-mediated pathogen detection and as a modulator of IFN levels [37]. In addition, TBK1 was an activator of the oncogenic AKT kinase and an activator of the KRASdriven cancer [38, 39]. Consequently, it seems that TBK1 was a linker of cancer pathogenesis and inflammation. Therefore, the role of TBK1 in PDAC requires further investigation.

In conclusion, we found low miR-429 level and high TBK1 level in PDAC promoted tumor cell growth, which contributed to low survival rate for patients with PDAC. MiR-429 exerted its role by targeting TBK1. We anticipate our results of our study provide invaluable information and provide a basis for further studies.

\section{Disclosure Statement}

The authors have declared that no competing interests exist. This work was supported by the grants from Natural Science Foundation of Shanghai, China (No. 12ZR1453900).

\section{Reference}

1 Song F, He M, Li H, Qian B, Wei Q, Zhang W, Chen K, Hao X: A cancer incidence survey in tianjin: The third largest city in china-between 1981 and 2000. Cancer Causes Control 2008;19:443-450.

-2 Yeo TP, Hruban RH, Leach SD, Wilentz RE, Sohn TA, Kern SE, Iacobuzio-Donahue CA, Maitra A, Goggins M, Canto MI, Abrams RA, Laheru D, Jaffee EM, Hidalgo M, Yeo CJ: Pancreatic cancer. Curr Probl Cancer 2002;26:176-275.

-3 Maitra A, Kern SE, Hruban RH: Molecular pathogenesis of pancreatic cancer. Best Pract Res Clin Gastroenterol 2006;20:211-226.

Ambros V: The functions of animal micrornas. Nature 2004;431:350-355. Garzon R, Calin GA, Croce CM: Micrornas in cancer. Annu Rev Med 2009;60:167-179.

Hou J, Lin L, Zhou W, Wang Z, Ding G, Dong Q, Qin L, Wu X, Zheng Y, Yang Y, Tian W, Zhang Q, Wang C, Zhuang SM, Zheng L, Liang A, Tao W, Cao X: Identification of mirnomes in human liver and hepatocellular carcinoma reveals mir-199a/b-3p as therapeutic target for hepatocellular carcinoma. Cancer Cell 2011;19:232-243.

-7 Li D, Liu X, Lin L, Hou J, Li N, Wang C, Wang P, Zhang Q, Zhang P, Zhou W, Wang Z, Ding G, Zhuang SM, Zheng L, Tao W, Cao X: Microrna-99a inhibits hepatocellular carcinoma growth and correlates with prognosis of patients with hepatocellular carcinoma. J Biol Chem 2011;286:36677-36685.

8 Mendell JT, Olson EN: Micrornas in stress signaling and human disease. Cell 2012;148:1172-1187.

9 Croce CM: Causes and consequences of microrna dysregulation in cancer. Nat Rev Genet 2009;10:704-714.

10 Park JY, Helm J, Coppola D, Kim D, Malafa M, Kim SJ: Micrornas in pancreatic ductal adenocarcinoma. World J Gastroenterol 2011;17:817-827.

11 Gregory PA, Bert AG, Paterson EL, Barry SC, Tsykin A, Farshid G, Vadas MA, Khew-Goodall Y, Goodall GJ: The mir-200 family and mir-205 regulate epithelial to mesenchymal transition by targeting zeb1 and sip1. Nat Cell Biol 2008;10:593-601.

12 Wellner U, Schubert J, Burk UC, Schmalhofer O, Zhu F, Sonntag A, Waldvogel B, Vannier C, Darling D, zur Hausen A, Brunton VG, Morton J, Sansom O, Schuler J, Stemmler MP, Herzberger C, Hopt U, Keck T, Brabletz S, Brabletz T: The emt-activator zeb1 promotes tumorigenicity by repressing stemness-inhibiting micrornas. Nat Cell Biol 2009;11:1487-1495.

13 Mees ST, Mardin WA, Wendel C, Baeumer N, Willscher E, Senninger N, Schleicher C, Colombo-Benkmann M, Haier J: Ep300--a mirna-regulated metastasis suppressor gene in ductal adenocarcinomas of the pancreas. Int J Cancer 2010;126:114-124.

14 Shen RR, Hahn WC: Emerging roles for the non-canonical ikks in cancer. Oncogene 2011;30:631-641.

15 Baldwin AS: Regulation of cell death and autophagy by ikk and nf-kappab: Critical mechanisms in immune function and cancer. Immunol Rev 2012;246:327-345.

16 Wu N, Liu C, Bai C, Han YP, Cho WC, Li Q: Over-expression of deubiquitinating enzyme usp14 in lung adenocarcinoma promotes proliferation through the accumulation of beta-catenin. Int J Mol Sci 2013;14:10749-10760. 
17 Wu N, Zhang C, Bai C, Han YP, Li Q: Mir-4782-3p inhibited non-small cell lung cancer growth via usp14. Cell Physiol Biochem 2014;33:457-467.

18 van Meerloo J, Kaspers GJ, Cloos J: Cell sensitivity assays: The mtt assay. Methods Mol Biol 2011;731:237245.

19 Han ZB, Yang Z, Chi Y, Zhang L, Wang Y, Ji Y, Wang J, Zhao H, Han ZC: Microrna-124 suppresses breast cancer cell growth and motility by targeting cd151. Cell Physiol Biochem 2013;31:823-832.

20 Sun T, Wang C, Xing J, Wu D: Mir-429 modulates the expression of c-myc in human gastric carcinoma cells. Eur J Cancer 2011;47:2552-2559.

21 Chien Y, Kim S, Bumeister R, Loo YM, Kwon SW, Johnson CL, Balakireva MG, Romeo Y, Kopelovich L, Gale M, Jr., Yeaman C, Camonis JH, Zhao Y, White MA: Ralb gtpase-mediated activation of the ikappab family kinase tbk1 couples innate immune signaling to tumor cell survival. Cell 2006;127:157-170.

22 Chariot A, Leonardi A, Muller J, Bonif M, Brown K, Siebenlist U: Association of the adaptor tank with the i kappa b kinase (ikk) regulator nemo connects ikk complexes with ikk epsilon and tbk1 kinases. The Journal of biological chemistry 2002;277:37029-37036.

23 Lewis BP, Burge CB, Bartel DP: Conserved seed pairing, often flanked by adenosines, indicates that thousands of human genes are microrna targets. Cell 2005;120:15-20.

24 Friedman RC, Farh KK, Burge CB, Bartel DP: Most mammalian mrnas are conserved targets of micrornas. Genome Res 2009;19:92-105.

25 Grimson A, Farh KK, Johnston WK, Garrett-Engele P, Lim LP, Bartel DP: Microrna targeting specificity in mammals: Determinants beyond seed pairing. Mol Cell 2007;27:91-105.

26 Garcia DM, Baek D, Shin C, Bell GW, Grimson A, Bartel DP: Weak seed-pairing stability and high target-site abundance decrease the proficiency of lsy- 6 and other micrornas. Nat Struct Mol Biol 2011;18:1139-1146.

27 Grentzmann G, Ingram JA, Kelly PJ, Gesteland RF, Atkins JF: A dual-luciferase reporter system for studying recoding signals. RNA 1998;4:479-486.

28 Reilly SM, Chiang SH, Decker SJ, Chang L, Uhm M, Larsen MJ, Rubin JR, Mowers J, White NM, Hochberg I, Downes M, Yu RT, Liddle C, Evans RM, Oh D, Li P, Olefsky JM, Saltiel AR: An inhibitor of the protein kinases tbk1 and ikk-varepsilon improves obesity-related metabolic dysfunctions in mice. Nature medicine 2013;19:313-321.

29 Lv T, Yuan D, Miao X, Lv Y, Zhan P, Shen X, Song Y: Over-expression of lsd1 promotes proliferation, migration and invasion in non-small cell lung cancer. PLoS One 2012;7:e35065.

-30 Kawai T, Akira S: Toll-like receptors and their crosstalk with other innate receptors in infection and immunity. Immunity 2011;34:637-650.

31 Calin GA, Croce CM: Microrna signatures in human cancers. Nat Rev Cancer 2006;6:857-866.

-32 Mattick JS, Makunin IV: Small regulatory rnas in mammals. Hum Mol Genet 2005;14 Spec No 1:R121-132.

-33 Tavazoie SF, Alarcon C, Oskarsson T, Padua D, Wang Q, Bos PD, Gerald WL, Massague J: Endogenous human micrornas that suppress breast cancer metastasis. Nature 2008;451:147-152.

34 Tie J, Fan D: Big roles of micrornas in tumorigenesis and tumor development. Histol Histopathol 2011;26:1353-1361.

-35 Uhlmann S, Zhang JD, Schwager A, Mannsperger H, Riazalhosseini Y, Burmester S, Ward A, Korf U, Wiemann S, Sahin O: Mir-200bc/429 cluster targets plcgamma1 and differentially regulates proliferation and egf-driven invasion than mir-200a/141 in breast cancer. Oncogene 2010;29:4297-4306.

-36 Chen J, Wang L, Matyunina LV, Hill CG, McDonald JF: Overexpression of mir-429 induces mesenchymal-toepithelial transition (met) in metastatic ovarian cancer cells. Gynecol Oncol 2011;121:200-205.

37 Ma X, Helgason E, Phung QT, Quan CL, Iyer RS, Lee MW, Bowman KK, Starovasnik MA, Dueber EC: Molecular basis of tank-binding kinase 1 activation by transautophosphorylation. Proc Natl Acad Sci U S A 2012;109:9378-9383.

-38 Chen H, Sun H, You F, Sun W, Zhou X, Chen L, Yang J, Wang Y, Tang H, Guan Y, Xia W, Gu J, Ishikawa H, Gutman D, Barber G, Qin Z, Jiang Z: Activation of stat6 by sting is critical for antiviral innate immunity. Cell 2011;147:436-446.

39 Barbie DA, Tamayo P, Boehm JS, Kim SY, Moody SE, Dunn IF, Schinzel AC, Sandy P, Meylan E, Scholl C, Frohling S, Chan EM, Sos ML, Michel K, Mermel C, Silver SJ, Weir BA, Reiling JH, Sheng Q Gupta PB, Wadlow RC, Le H, Hoersch S, Wittner BS, Ramaswamy S, Livingston DM, Sabatini DM, Meyerson M, Thomas RK, Lander ES, Mesirov JP, Root DE, Gilliland DG, Jacks T, Hahn WC: Systematic rna interference reveals that oncogenic kras-driven cancers require tbk1. Nature 2009;462:108-112. 